\title{
Perspectiva del doctor Jorge Chancos: Apreciación, aportes e influencias a la geografía peruana
}

\author{
Dr. Jorge Chancos' perspective: Appreciation, contributions, and influences \\ to Peruvian geography
}

\section{Perspetiva do Dr. Jorge Chancos: Valorização, contribuições e influências para a geografia peruana}

\author{
Angie Dayana Condori Lazo y Bruno Jahiro Pintado Caparachin ${ }^{b}$ \\ Universidad Nacional Mayor de San Marcos \\ a angiedayana.condori@unmsm.edu.pe, Código ORCID: https://orcid.org/0000-0002-2112-9191 \\ b bruno.pintado@unmsm.edu.pe, Código ORCID: https://orcid.org/0000-0002-3877-3901
}

\begin{abstract}
RESUMEN
Jorge Chancos Pillaca es un reconocido geógrafo peruano. Nació en el distrito de Huancapi, provincia de Fajardo, Ayacucho en 1937. Es autor de grandes investigaciones plasmadas en sus tesis siendo una de las más relevantes su tesis doctoral: Deshielo de los glaciares de la Cordillera Pariaqaqa y su influencia en el origen y caudal del rio Rímac (2011) auspiciados por la Sociedad Geográfica de Lima. Además de su último libro más destacable Teoría de la Geografía y geógrafo (2019) donde desarrolla el pensamiento geográfico a lo largo del tiempo. En esta entrevista, dirigida a todo público nacional y extranjero interesado en conocer la evolución del pensamiento geográfico del Perú, Jorge Chancos Pillaca nos detalla distintos aspectos de la geografía peruana resaltando el pensamiento geográfico desde la época del Tawantinsuyo. Da a conocer sus inicios en la Geografía en la Decana de América, brinda una definición de la geografía, inclusive los geógrafos que influyeron en su formación, destacando el hecho de conocer a Milton Santos; asimismo explica la razón por la que se inclino a realizar estudios de cuencas, su opinión respecto a los cambios en el modelado del territorio peruano y nos detalla algunos capítulos de su gran libro "Teoría de la geografía y geógrafos", concluyendo con una opinión respecto a lo importante que es el geógrafo debido a su carácter multidisciplinar e integrador.
\end{abstract}

\begin{abstract}
Jorge Chancos Pillaca is a renowned Peruvian geographer. He was born in huancapi district, Fajardo province, Ayacucho in 1937. He is the author of great research embodied in his thesis is one of the most relevant his doctoral thesis: Melting the glaciers of the Pariaqaqa Range and their influence on the origin and flow of the Rimac River (2011) sponsored by the Lima Geographical Society. In addition to his latest most notable book Theory of Geography and Geographer (2019) where he develops geographical thinking over time. In this interview, aimed at every national and foreign public interested in learning about the evolution of Peru's geographical thinking, Jorge Chancos Pillaca details different aspects of Peruvian geography highlighting geographical thinking since the time of Tawantinsuyo. It makes known its beginnings in Geography in the Dean of America, provides a definition of geography, including geographers who influenced its formation, highlighting the fact that we met Milton Santos; it also explains why I am inclined to conduct watershed studies, his opinion regarding the changes
\end{abstract}


in the modeling of the Peruvian territory and details some chapters of his great book "Theory of Geography and Geographers", concluding with an opinion on how important the geographer is due to its multidisciplinary and integrative nature.

\section{RESUMO}

Jorge Chancos Pillaca é um renomado geógrafo peruano. Ele nasceu no distrito de Huancapi, província de Fajardo, Ayacucho em 1937. Ele é autor de grande pesquisa incorporada em sua tese sendo uma das mais relevantes sua tese de doutorado: Degelo das geleiras da Cordilheira Pariaqaqa e sua influência na origem e vazão do rio Rímac (2011) patrocinada pela Sociedad Geográfica de Lima. Além de seu mais recente livro Teoria da Geografia e Geógrafa (2019), onde desenvolve o pensamento geográfico ao longo do tempo. Nesta entrevista, voltada para todos os públicos nacionais e estrangeiros interessados em conhecer a evolução do pensamento geográfico do Peru, Jorge Chancos Pillaca detalha diferentes aspectos da geografia peruana destacando o pensamento geográfico desde a época do Tawantinsuyo. Faz com que seu início em Geografia na América, forneça uma definição de geografia, incluindo geógrafos que influenciaram sua formação, destacando o fato de conhecer Milton Santos; também explica por que ele estava inclinado a realizar estudos de bacias, sua opinião sobre as mudanças na modelagem do território peruano e detalha alguns capítulos de seu grande livro "Teoria da Geografia e Geógrafos", concluindo com uma opinião sobre a importância do geógrafo devido ao seu caráter multidisciplinar e integrativo.

PALABRAS CLAVES: Pensamiento geográfico; espacio; perspectiva; cuenca; territorio.

KEYWORDS: Geographic thinking; space; perspective; basin; territory.

PALAVRAS-CHAVE: Pensamento geográfico; espaço; perspectiva; bacia; território.

Angie Condori (AC): Realizamos una recopilación de información allegadas a diversas fuentes relacionadas a su formación como geógrafo en las diversas universidades de las cuales adquirió conocimientos, ante ello planteamos, ¿Por qué decidió optar por esta carrera? A raíz de esta pregunta, ¿Cómo podría usted definir a la geografía?

Jorge Chancos (JC) : En primer término, agradezco la gentileza de invitarme para participar en la Revista Espiral; al mismo tiempo, felicito a la nueva generación de estudiantes de Geografía de la cuatricentenaria Universidad Nacional Mayor de San Marcos por la brillante iniciativa de crear un órgano de difusión para relanzar opiniones y corrientes de pensamiento de Geografía en el marco del bicentenario nacional.

Respondiendo a su pregunta inicial. El hecho de haber seguido la carrera de geografía es anecdótico. A finales de 1960, los dos primeros años se hacía Estudios Generales en Letras o Ciencias. Postulé para seguir sociología, porque era una carrera nueva. En Letras estábamos juntos con los estudiantes de: psicología, sociología, educación, historia, economía, contabilidad, derecho, antropología y geografía; las aulas rebalsaban y los profesores usaban micrófonos. El catedrático de sociología no me convenció con sus clases para continuar en sociología; el profesor de geografía era el Dr. Ciro Hurtado Fuertes, sus clases eran amenas, didácticas; salíamos a las clases en el campo. Recuerdo, la visita a la central hidroeléctrica de Huinco la casa de máquinas de cuatro pisos estaba a dentro de la montaña, en la margen izquierda del río Santa Eulalia. La movilidad, guía y almuerzo de gala fue ofrecido por la empresa, me impresionó tanto que me enamoré de Geografía. El Dr. Ciro Hurtado Fuertes fue quien influyó para cambiarme de sociología a la especialidad de Geografía.

Hasta ahora sigue la indefinición de la geografía, hay tantas definiciones como geógrafos existe. La tradicional pregunta ¿qué es Geografía?, sigue siendo vigente. Todas las ciencias estudian algún aspecto del planeta Tierra. Las Ciencias Sociales, como: Historia, antropología, arqueología, economía, sociología y psicología tienen objeto de estudio específico de algún aspecto del ser humano. La geografía es la Ciencia Social, cuyo objeto de estudio es el hombre o la sociedad en relación e 
interacción permanente con los elementos de su contexto espacio-temporal. Por su naturaleza la geografía es interdisciplinar y transdisciplinar, de visión horizontal de la totalidad del espacio como una unidad orgánica a escala local, regional o global. La geografía no estudia un espacio abstracto; sino, el espacio social construido por el hombre; es decir, el espacio históricamente ocupado, vivido, modificado y reordenado según su concepción del mundo, su ideología, avances científicos y tecnológicos. Por ejemplo, cuando ocuparon los primeros hombres en Piqui Machay-Ayacucho, sucesivamente fueron ocupados el mismo espacio social por los waris, chancas, inkas, colonos españoles y las generaciones de la vida republicana; cada cultura reordenaba su espacio en cada periodo de tiempo que les tocó vivir.

Bruno Pintado (BP): De acuerdo a su gran trayectoria y experiencia en eventos, ponencias e investigaciones internacionales ¿Qué geógrafos fueron de mayor influencia para su desarrollo? Es relevante mencionar al geógrafo brasileño Milton Santos por sus grandes aportes en contextos contemporáneos al de usted ¿Considera que influyo en sus ideas y por qué?

(JC): La influencia en nuestras ideas, manera de pensar y actuar se adquiere más, con la lectura de las obras y su mensaje. En este sentido, los geógrafos que de alguna manera influyeron en mi formación profesional, fueron: Pierre George, Yves Lacoste, Oliver Dollfus, David Harvey, Horacio Capel, Milton Santos, Ruy Moreira. Entre los nacionales no puedo dejar de reconocer, a: Pulgar Vidal, Peñaherrera, Ciro Hurtado, Juan La Cruz López; quienes mediante sus publicaciones dieron pautas en el devenir de la geografía.

Tratar de la persona de Milton Santos es relevante, por ser el geógrafo iberoamericano de talla mundial, reconocido por el mundo intelectual contemporáneo. En el periodo de la dictadura, Milton Santos fue desterrado, tiempo que aprovechó para visitar y dictar conferencias en las universidades principales de Europa, Asia, África y América Latina. Como homenaje en vida a Milton Santos, los geógrafos brasileños en colaboración con los geógrafos del mundo, escribieron y publicaron el libro titulado "El mundo del ciudadano y ciudadano del mundo" (1996), en la que valoran a Milton Santos: como persona, político, académico, de sus obras magistrales y de la Geografía Nueva brasileña. Otra publicación en homenaje póstumo al maestro Milton es la obra titulada "Tras las huellas de Milton Santos Una mirada latinoamericana a la geografía humana contemporánea" (2008), organizado por los geógrafos mexicanos y brasileños, bajo la coordinación del Dr. Cristóbal Mendoza de la Universidad Autónoma MetropolitanaIztapalaca de México.

Tuve el honor de conocer personalmente a Milton Santos, en junio de 1997, cuando fui con Ciro Hurtado al "I ENCONTRO DE GEOGRAFIA DA BAHIA. OS LUGARES DO MUNDO. A GLOBALIZAÇÃO DOS LUGARES". Sus principales obras como: Por una Geografía Nueva (1990), Técnica Espaço Tempo Globalização e medio técnico científico informacional (1997) y La naturaleza del espacio Técnica y tiempo, Razón y emoción (2000), tratan de temas novedosas como: "La Geografía viuda del espacio", "la dimensión histórico temporal y la noción de totalidad", "El espacio sistema de objetos y sistema de acciones" y muchísimos otros asuntos de carácter epistemológico; así como, de las tendencias y corrientes del pensamiento geográfico. En este sentido, me ha permitido reflexionar y revalorar al espacio social, escenario cotidiano de la sociedad.

(AC): En su perfil profesional como geógrafo destaca en grandes aspectos su interés por los estudios e investigaciones relacionadas a cuencas y ríos, en virtud de ello, ¿Por qué se inclinó a realizar estudios en base a estas áreas? Rescatando lo anterior, ¿Cuáles fueron las utilidades y dificultades de dichos estudios?

(JC): Es muy simple, observar la concentración y distribución demográfica que están a lo largo de un río, en el litoral de los lagos y mares. Los hechos sociales, 
culturales, la actividad económica; así como, los ecosistemas y los eventos naturales ocurren dentro de una cuenca hidrográfica. Absolutamente nada está fuera de una cuenca; después de todo, la red hidrográfica es la que determina el tamaño de una cuenca (mega cuenca, macro cuenca, meso cuenca y mini cuenca, no existe "micocuenca", habría que observar con microscopio); por consiguiente, los problemas sociales, económicos, políticos y ambientales están en dichas áreas. Por ejemplo, un problema vial casi frecuente se presenta cuando hay un deslizamiento o flujo torrencial (lloqlla, no Wayqo) que interrumpe la circulación normal de transportes; éste fenómeno, deriva múltiples impactos: ambientales, económicos, sociales, pérdida de vidas humanas, pérdida de horas hombre, encarecimiento de productos, pérdida de productos perecibles, y una cadena de secuencias; eventos que afectan a toda la población de la cuenca donde se produjo. Razones más que suficientes para considerar a la cuenca como unidad espacial de gestión administrativo ambiental. La utilidad de una investigación depende de la relevancia y alcances que tenga; la dificultad radica en el financiamiento y apoyo institucional para realizar investigaciones trascendentales.

(BP): Recopilando información sobre su hoja de vida (estudios, proyectos e investigaciones) es preciso recalcar la gran variedad de aportes que ha brindado a lo largo de los años. Respecto a ello, ¿Cuál considera ser el más importante que ha realizado, en qué contexto lo hizo y qué lo impulsó?

(JC): A parte de mi tesis doctoral sobre El deshielo de los glaciares de la cordillera Pariaqaqa y su influencia en el origen y caudal del río Rímac (2011), cuya publicación ha sido auspiciada por la Sociedad Geográfica de Lima. Considero de mayor trascendencia mi reciente publicación, Teoría de la Geografía y Geógrafos (2019), un aporte específico a la Geografía. Realicé durante el ejercicio de la docencia en la Universidad Nacional del Callao (UNAC) y la Universidad Nacional Enrique Guzmán y Valle (UNE-EGV), entre 1992 y 2017. Los motivos surgieron anecdóticamente

1. En la UNAC, había una asignatura "Pensamiento Económico", repensando dije, por qué no puede haber ¿pensamiento geográfico?, abordaría sobre la evolución histórica de la geografía. Un día en una librería encontré el libro de Josefina Gómez Mendoza, con el título "El pensamiento geográfico" (1982).

2. En los años 1985, con la Ley universitaria 17437, culminado el octavo ciclo los estudiantes podrían obtener el grado académico de Bachiller dando un examen de conocimiento. Un grupo de diez estudiantes se presentaron al examen, una de las preguntas que formulamos era ¿Qué es Geografía? Ninguno respondió.

3. Un alumno de geografía al salir de la clase de filosofía airadamente me aborda diciendo, "profesor, ¿es verdad que la geografía no es ciencia?; entonces, para qué estoy estudiando". Esto, motivó una mesa redonda de la plana docente para explicar a todos los alumnos sobre el carácter científico de la Geografía; parece que no le convencimos; el aludido estudiante, en el siguiente ciclo se trasladó a la especialidad de Historia.

Estos tres actos me hicieron reflexionar de la carencia del manejo epistemológico doctrinario de geografía. Para solucionar esta deficiencia, creamos la asignatura Pensamiento Geográfico en el plan curricular de geografía de 1987 de la UNE. Enrique Guzmán y Valle. Luego, asumí la cátedra de esta asignatura. Cuando se creó la maestría en Ciencias de la Educación-mención Geografía en la UNE-EGV. en 1988, esta asignatura formó parte del Plan de estudios con el nombre de Teoría de la Geografía, el Dr. Hurtado se hizo cargo de la asignatura. Desde 1997, en pregrado de la especialidad de Geografía de la UNE se cambió Pensamiento Geográfico por Teoría de la Geografía hasta ahora. Años después, se creó esta asignatura en la Universidad 
Nacional Mayor de San Marcos; así como, en la Escuela de post grado de Ciencias Histórico Sociales de la Universidad de San Agustín de Arequipa.

Es así, en el fragor del debate en las clases con estudiantes de pre grado y post grado mejoré el sílabo ciclo tras ciclo; luego desarrollé el texto TEORÍA DE LA GEOGRAFÍA Y GEÓGRAFOS de manera didáctica, con un lenguaje coloquial, dirigido a los estudiantes de la especialidad de geografía y ciencias sociales y, a los profesores de Educación Básica. Espero la buena acogida por parte del lector amante de la geografía.

(AC): El Perú es un país con un gran geosistema ya que posee distintos elementos tanto Bióticos, Abióticos como Antrópicos, que, a través del tiempo, muchos de estos han variado generando diversos impactos. Ante ello, ¿Cuál es opinión respecto a estos cambios del modelado del territorio peruano en el marco del cambio climático?

(JC): El modelado del territorio peruano ha sido un proceso que duró millones de años; la última fase tectónica de la orogenia "plegamiento andino" ocurrió en el mioceno medio a superior del periodo terciario, alcanzando sus niveles y geo formas actuales. Durante el pleistoceno y holoceno del cuartiario se produce la degradación por la erosión de los agentes de la geodinámica externa. Se sabe, que durante el periodo cuartiario se ha producido 4 glaciaciones y desglaciaciones; que no es otra cosa, sino, los grandes cambios climáticos. El reciente recalentamiento global, producto de la alteración de los gases del efecto invernadero está originando cambios climáticos con secuelas múltiples de orden geofísico, biológico y humano., que puede estar reajustando el relieve local. Frente a estos eventos lo que cabe al hombre es prevenir y educar a la población (hablar de educación geográfica, no "educación ambiental") para mitigar sus efectos perversos.

Producto del modelado del suelo peruano contemporáneo, el Perú es un país ribereño ubicado frente al mar más rico del mundo; un país andino polimetálico y multiclimático; un país amazónico de gran biodiversidad y pulmón del mundo. Un país heredero de una cultura avanzada; esas son nuestras fortalezas; la gran debilidad está en la sociedad, con una democracia incipiente, una clase política inmadura, ambiciosa y corrupta; las amenazas son de orden físico natural como: el cambio climático y sus efectos: sismos, maremotos, plagas como el "covid-19"; y de orden social como: las guerras, convulsiones sociales, terrorismo, etc. Sin embargo, toda crisis da la oportunidad de dar saltos cualitativos en el orden científico, tecnológico y social. Esperamos que los gobernantes políticos reflexionen y rectifiquen su conducta y mejoren las condiciones ambientales para vivir humanamente.

(BP): Uno de sus últimos libros con un mayor grado de popularidad es "Teoría de la geografía y geógrafos", publicado el 2019, donde el capítulo II abarca el pensamiento geográfico a través del tiempo. Una interrogante necesaria es ¿Cómo ha variado el pensamiento geográfico en el Perú y en qué contexto hace mayor referencia en su libro? ¿Considera usted que el pensamiento geográfico peruano existe o es una adaptación del extranjero de las escuelas de los siglos 19 y 20 ?

(JC): Entendiendo la geografía como ciencia social que estudia al hombre en estrecha relación e interacción con cada uno de los elementos del geosistema, se puede afirmar a la luz de los hechos emblemáticos que la cultura Tawantinsuyana organizó el espacio social de manera racional. Sin conocer la escritura, creando su propia tecnología y metodología del manejo de cuencas hidrográficas realizaron el trasvase de cuencas, construyeron acueductos, reservorios y sistema de andenes; emplazaron los asentamientos humanos en lugares estratégicos, previniendo contra los fenómenos naturales y la seguridad física ciudadana. De la misma manera, fueron los que construyeron las obras de infraestructura con el verdadero pensamiento 
de "desarrollo sostenible", obras que hasta ahora son duraderas para el disfrute de muchas generaciones. El cambio de pensamiento geográfico se produce con el cambio de las relaciones sociales y el modo de producción del periodo colonial español; como resultado se produjo una involución del desarrollo de la geografía.

En la vida republicana el pensamiento geográfico ha ido cambiando conforme va influyendo la geografía moderna y la geografía nueva contemporánea y de acuerdo a la institucionalización académica en la educación básica regular y en las universidades. La crisis de la geografía como ciencia se origina con la desaparición del plan curricular nacional en educación básica regular; al ser fusionando en el área de Ciencias Sociales con horas mínimas de clase; en la mayoría de las carreras públicas no se enseña geografía. Los avances, estancamiento o retroceso del pensamiento geográfico lo abordamos en el contexto de la evolución histórica del pensamiento geográfico en el Perú durante los periodos: prehispánico, virreinato y la república.

A tu pregunta si existe o no, el pensamiento geográfico en el Perú. Te respondo, que sí; como humanos somos seres pensantes, claro, no todos pensamos igual. En el párrafo anterior demostramos cómo los Inkas pensaban de la relación del hombre (runa) con la Mama Pacha (Tierra madre), ellos respetaban a la naturaleza y mantenían un equilibrio ecológico. Cada geógrafo tiene su propio pensamiento de acuerdo a su ideología; lo que sucede, es que no hay una Escuela Geográfica peruana, ni tendencia, ni corriente de pensamiento colectivo; cada uno, adopta la corriente de pensamiento geográfico conforme lee y se informa de las bondades de cada corriente desarrollada por la Escuela: alemana, francesa, anglosajona, norteamericana, rusa u otras. Me atrevería considerar, que hay un germen de la corriente sistémica de la geografía peruana, promovida por el Dr. Ciro Hurtado Fuertes desde los años 1970, seguido por algunos de sus discípulos quienes publican sus obras con ese enfoque; si otros geógrafos aplicaran este enfoque como método e instrumento de análisis de los problemas geográficos, estaríamos diciendo que existe la corriente de pensamiento geográfico en el Perú.

(AC): En el capítulo VI del libro aludido engloba con una mayor profundidad las corrientes, tendencias y perspectivas del pensamiento geográfico en un marco definido. Desde su criterio, ¿Qué corriente, tendencia y perspectiva predomina en el desarrollo de su tesis de Bachiller "Bases geográficas para el cultivo de trucha en los lagos de la cuenca alta del río Cañete - 1974"?

(JC): Buena pregunta, en esa época no existía asignaturas de investigación que conlleve a desarrollar una tesis; entonces lo realizábamos sin ningún rigor científico. Actualmente, hay normas que exigen: Planteamiento del problema, antecedentes, fundamentación, hipótesis, métodos, variables, etc. El título de mi investigación fue producto de una salida al campo aprovechando días festivos, en compañía del Dr. Ciro Hurtado, Bertha Balbín, César Soto y Juan Vilchez en 1970. Observar los hermosos paisajes alto andinos matizados de glaciares, lagos en rosario en el fondo de los valles secundarios y del principal, ríos de aguas cristalinas intercaladas con cascadas, las estancias de pastores de ovejas, llamas y ganado vacuno esparcidas en la puna de la cuenca alta del río Guarco o Cañete, me fascinó. En voz alta dije a mis compañeros: de este lugar hago mi tesis de bachillerato; intercambiando ideas, surgió el título Bases geográficas para el cultivo de la trucha en los lagos de la cuenca alta del río Cañete.

En esos años, la tendencia de la geografía regionalista era conocida en nuestro medio por la obra de Javier Pulgar Vidal y la tendencia cuantitativa de la geografía estaba de moda; pero, no teníamos noción clara de estas tendencias. Por un elemental razonamiento lógico había que identificar en el espacio la realidad material del área de estudio, precisando los factores concurrentes para la crianza de la trucha; así mismo, identificar los problemas socio económicos de la población alto andina. Para conocer los atributos geofísicos ambientales se ha aplicado el positivismo y el 
empirismo materialista de Francis Bacon, mediante el método de observación directa, aplicación de la tecnología cartográfica y foto lectura. En el trabajo de investigación se ha relacionado: espacio, tiempo, técnica y materia, que son categorías universales; en cierto modo, se aplicó aspectos de tendencia regionalista y cuantitativa de la geografía; y, la corriente del pensamiento racionalista andina.

Siendo un tema transversal, integradora de visión holística y de una geografía aplicada, la perspectiva fue difundir y propiciar el pragmatismo a fin de motivar a resolver problemas alimenticios, laborales y económicos de la población mediante el cultivo intensivo de la trucha "arco iris" en piscigranjas: comunales, cooperativas, escolares y familiares. Sobre el tema, di algunas charlas en la radio Comas, a solicitud del Centro Social Huaros residentes en Lima, quienes estaban interesados en construir una piscigranja. Ellos, concretaron su proyecto y fue la primera "piscigranja de la comunidad campesina de Huaros-Canta"; hoy, es una empresa comunal que da trabajo a sus hijos, alimenta a la comunidad y comercializa con algún mercado limeño.

La tesis fue publicada en 1978, la librería Studium, la Familia y Época comercializaron; los consumidores fueron los estudiantes de la Universidad Federico Villarreal y Agraria La Molina donde tenían Facultad de pesquería y de pesca continental. Ahora hay piscigranjas a lo largo y ancho del mundo andino.

(BP): Otro de los capítulos de gran interés en su libro ya mencionado es el capítulo V donde informa sobre la historia de la geografía en Perú, ¿Nos podría detallar más sobre cómo ha ido evolucionando esta carrera en San Marcos? (Inicios)

(JC): Antes de la creación del Instituto de Geografía en 1947, la Geografía como ciencia fue incorporada en la UNMSM, por Bartolomé Herrera en 1845. Desde esa época surge la tendencia de la geografía física y la tendencia de la geografía humana, hasta ahora continúa esta dicotomía. La geografía como ciencia es una sola. La Geografía moderna en el Perú, fue impulsada por el científico naturalista Antonio Raimondi (1826-1890) y por las expediciones científicas y personajes han visitado al Perú. Los precursores de la Geografía contemporánea a mi parecer fueron; Mateo Paz Soldán y Ureta, con su obra Geografía del Perú (1862); Mariano Felipe Paz Soldán y Ureta, con la publicación Atlas Geográfico del Perú y Diccionario Geográfico Estadístico del Perú (1965); Germán Stiglich, con muchas obras, como Geografía Elemental Ilustrada (1914), Diccionario Geográfico del Perú (1922), Carlos Nicholson, quien ha cartografiado el mapa climático del Perú en base a la clasificación climática de Köppen; Oscar Miroquesada de la Guerra, con su obra Geografía Científica del Perú (1919).

La Geografía en el año 1946, se enseñaba en varias Facultades: Geografía Física y Matemática, en la Facultad de Ciencias; Geografía Económica, en la Facultad de Economía; Geografía Humana, en la Facultad de Letras; y Métodos de Enseñanza de la Geografía, en la Facultad de Educación.

La publicación de la magnífica obra Las Ocho Regiones Naturales del Perú de Javier Pulgar Vidal, tuvo un impacto científico que cambió la visión tradicional del Perú, hecho que permitió la creación del Instituto de Geografía en 1947 para desarrollar la carrera de Geografía propiamente dicha. Los profesores eran extranjeros, mayormente franceses. Las primeras promociones crearon la Asociación de Egresados del Instituto de Geografía de la Universidad Nacional Mayor de San Marcos; luego, organizaron el Primer Congreso Nacional de Geografía realizado en Otuzco, donde fundaron la Asociación Nacional de Geógrafos Peruanos, el 3 de agosto de 1961, eligiendo su Junta Directiva. El II congreso se realizó en Arequipa en 1964 y el tercer Congreso de Geografía se llevó acabo en Iquitos 1966. Las ponencias de temas importantes de diversos aspectos fueron editadas y publicadas en varios textos: Geografía Humana General (1962), Geografía de los Recursos Naturales Vegetales (1962), El mar del Perú es una región geográfica (1966), 
Geografía Humana, Económica y Política (1966), Geografía Física (1967), Geografía Humana (1967) y Geografía regional (1967). Considero que fue la época de oro de la producción intelectual de geografía en el Perú.

Los integrantes de la Junta Directiva fueron: Heráclides Vergaray (Presidente), Ciro Hurtado, Efraín Orbegoso, Pedro Herencia, Ernesto Curril, Jaime Rivera, Mario Escobar, Teodoro Casana, Juan Urbina, César Cabrera, Oscar Santillán, Juan Benavides Estrada, Alberto Andrade, entre muchos otros. Algunos de ellos docentes de la UNMSM:

Los egresados de fines 1960 e inicios del 70, ya no participamos de la Asociación de Geógrafos Peruanos, ya había desaparecido. Siempre hay, entusiasmo e inquietud en los estudiantes para difundir que existe Geografía como especialidad en la UNMSM., así aparece la Revista EL GEÓGRAFO, que publicó dos números con temas igualmente interesantes, propiciado por el Centro de Estudiantes de Geografía.

Con los egresados de 1969, 70, 71 y 72, se creó el Centro de Investigaciones Geográficas (CIG), que tuvo vigencia de once años, publicándose 5 números de CUADERNOS DE GEOGRAFÍA; una edición especial de la obra de Jean Paul Deler, titulado Aspectos del crecimiento de la capital peruana 1940-1970 (1975); otra obra, Geografía del subdesarrollo de Yves Lacoste (1976), traducido al castellano por Luis Oliveros Lakoma. Finalmente, hasta donde recuerdo en 1983 se creó el LABORATORIO DE ESTUDIOS GEOGRÁFICOS EN PLANIFICACIÓN RURAL Y URBANA, editando el Boletín N ${ }^{\circ}$ 1, en 1983.

Entre los autores frecuentes de los artículos científicos en las publicaciones mencionadas podemos mencionar a: Hildegardo Córdova Aguilar, Bertha Balbín, Jorge Arnao, Alfredo Giraldo; Ángel Rojas, Luis Alberto Oliveros, Jorge Chancos, Zoila Rodríguez, Walter Aguilar, Florencio Guzmán M., Francisco Huamán; entre los docentes: Pulgar Vidal, Ernesto Curril, Alberto Mc, Key, Nicole Bernex de Falen. Para que tengan idea de cómo ha ido evolucionando el pensamiento geográfico en el Perú les invito revisar las publicaciones mencionadas.

(AC): La trayectoria de la geografía en la Decana de América, ha destacado por sus diversos aportes a las Ciencias Sociales desde contextos muy remotos. Sin embargo, también se presentaron pequeños obstáculos para el desarrollo práctico de la carrera. ¿Qué recuerda con frecuencia de la carrera de geografía en San Marcos qué lo haya diferenciado de otras casas de estudio?

(JC): Es verdad, hubo grandes personajes en todas las áreas profesionales formados en la Universidad Nacional Mayor de San Marcos (UNMSM) desde su fundación en 1551 quienes contribuyeron en el desarrollo del país. La carrera de geografía recién se crea a mediados del siglo pasado en UNMSM; y, en la Pontificia Universidad Católica del Perú en 1987. Son las únicas universidades que forman geógrafos. No hay otra casa de estudios que forme geógrafos para diferenciarlos.

El problema en el Perú, como en otras universidades de América Latina ha sido y sigue siendo la carencia del dominio epistemológico, la ambigüedad y la indefinición del objeto de estudio de la Geografía. En San Marcos, al no saber exactamente qué es Geografía, a veces está en el área de Ciencias Sociales, otras veces está en Ciencias naturales junto con Geología, metalurgia, minas y mecánica de fluidos. En Chile está en la Facultad de Arquitectura; en Argentina y México, está en la Facultad de Humanidades; en Cuba, la Geografía es una Facultad independiente; en la universidad de Sao Paulo, está en la Facultad de Filosofía y Ciencias; y así, no hay uniformidad académica, estructural ni institucional en América Latina ni en el mundo. Esta incertidumbre ha originado la creación de Ingeniería Geográfica, tanto en Villareal como en San Marcos. La diferencia entre una y otra universidad en cuanto a los 
geógrafos está en la producción intelectual y participación en las actividades sociales, políticas y culturales del país.

(BP): Es fundamental reconocer su gran trayectoria y aportes que ha hecho usted a la Geografía, agradecemos mucho su tiempo y dedicación. En una forma de conclusión, ¿Qué tan importante considera que es el geógrafo para el Perú?

(JC): El Perú con grandes potencialidades de recursos naturales y culturales, con una sociedad plurilingüe y multiétnico, requiere la participación de equipos multidisciplinares para recoger información de campo y plantear soluciones integrales de los problemas tan complejos de carácter, económico, político, cultural y de ordenamiento territorial. Desde este punto de vista, la participación del geógrafo por su carácter multidisciplinar e integrador es muy importante. El geógrafo conoce los atributos geofísicos del territorio: relieve, clima, suelo, hidrografía y ecosistemas; del mismo modo, conoce a la población: su estructura, dinámica, sus actividades económicas y problemas sociales tanto urbanos como rurales y el deterioro ambiental. Con la visión sistémica, holística y transversal de los lugares, con el manejo tecnológico del sistema de información geográfica (SIG), está en la capacidad de asumir la dirección de proyectos y formular soluciones integrales.

ElColegio de Geógrafos del Perú(CGP), debetener presencia activa y permanentemente en el Estado, opinando y proponiendo alternativas de solución a los problemas sociales y Geoambientales. A manera de reflexión opino, el CGP., tiene la oportunidad de plantear la solución de: a) La actual regionalización o departamentalización trae muchos problemas limítrofes, burocráticos y trabas administrativas, es necesario un nuevo ordenamiento territorial respetando las cuencas hidrográficas como unidades de gestión ambiental. b) La Geografía ha sido minimizada o casi desaparecida del sistema educativo, cabe gestionar ante el Ministerio de Educación la reincorporación de la Geografía como asignatura independiente en la malla curricular de Educación Básica Regular, en los Institutos Superiores y en las carreras universitarias para crear conciencia de peruanidad y amor topofílico. c) Propiciar que la Escuela Profesional de Geografía de la UNMSM se convierta en una Facultad. Muchas gracias señores estudiantes, les auguro nuevos rumbos y mejores perspectivas en base a la lectura, investigación y publicación.

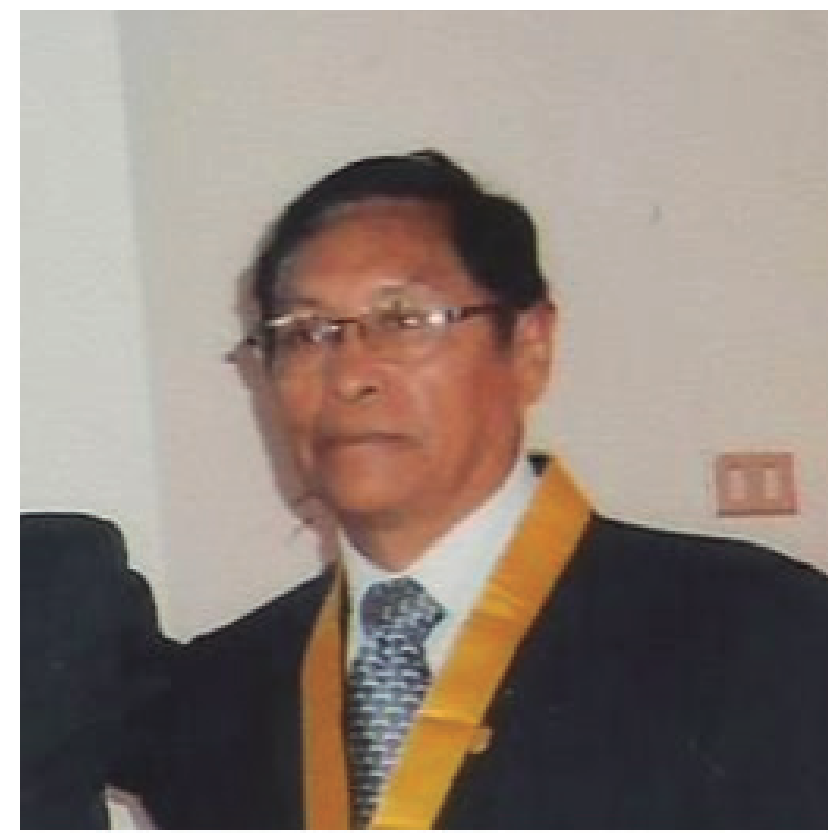

Jorge Chancos Pillaca 
